\title{
Outcome of Cataract Surgery in Patients Treated for Retinopathy of Prematurity
}

\author{
Huy Nguyen', Kimberly G. Yen ${ }^{1,2}$ \\ ${ }^{1}$ Department of Ophthalmology, Baylor College of Medicine, Houston, TX, USA \\ ${ }^{2}$ Department of Ophthalmology and Pediatrics, Texas Children's Hospital, Houston, TX, USA \\ Email: kgyen@texaschildrens.org
}

How to cite this paper: Nguyen, $H$. and Yen, K.G. (2017) Outcome of Cataract Surgery in Patients Treated for Retinopathy of Prematurity. Open Journal of Ophthalmology, 7, 293-302.

https://doi.org/10.4236/ojoph.2017.74038

Received: September 25, 2017

Accepted: November 3, 2017

Published: November 6, 2017

Copyright (c) 2017 by authors and Scientific Research Publishing Inc. This work is licensed under the Creative Commons Attribution International License (CC BY 4.0).

http://creativecommons.org/licenses/by/4.0/

(c) (i) Open Access

\begin{abstract}
Background/Aims: Pediatric patients with treated retinopathy of prematurity (ROP) may develop visually significant cataracts. We report the outcome of cataract surgery in patients who had ROP treatment. Method: Retrospective chart review of 19 eyes from 16 patients who had ROP treatment and subsequent cataract surgery between August, 2002 and March, 2015. Results: Eighteen of 19 eyes received laser treatment for ROP; 1 eye received intravitreal bevacizumab. 5 eyes received lens-sparing pars plana vitrectomy (LSPPV) in addition to laser. Average follow up was $10.1 \pm 5.5$ years. Average visual acuity improved from 20/324 prior to and 20/110 after cataract surgery $(\mathrm{p}=0.06)$. $13 / 19(68 \%)$ of the eyes received laser only and developed cataracts an average of $6.2 \pm 5.6$ years after laser treatment. 5/19 (26\%) eyes developed cataracts an average of $6.4 \pm 4.2$ years after LSPPV and laser. In one eye, a cataract developed after a bevacizumab injection 2.9 years after the injection. Visual axis opacification (VAO) developed in 2/5 (40\%) eyes after Ce/PCIOL/PPC-Antvx, $8 / 10$ eyes (80\%) after CE/IOL, and in 0/4 eyes after CE/PPC-AntVx. Ocular comorbidities included strabismus, nystagmus, amblyopia, optic atrophy, corneal band keratopathy, and phthisis bulbi. Conclusion: Cataract surgery in patients who have a history of ROP can be complicated by anatomical changes from prematurity and prior vitreoretinal surgeries. Vision improvement is limited by other ocular comorbidities.
\end{abstract}

\section{Keywords}

Pediatric Cataract, Retinopathy of Prematurity, Pediatric Cataract Surgery

\section{Introduction}

Patients treated for retinopathy of prematurity (ROP) may suffer limited vision 
from amblyopia, anatomic retinal changes, and glaucoma [1]. Cataracts occur frequently in this high risk population [2] [3] and cataract surgery may be complicated due to history of previous vitreoretinal surgery or ocular changes due to the ROP. Postoperative management may present additional challenges due to refractive changes over time, visual axis opacification (VAO), and anatomic retinal changes [4] [5] [6] [7].

The etiology of cataracts in patients treated with ROP laser is unclear. Studies have evaluated the possibility of anterior segment ischemia as a potential cause and have not found that earlier treatment for the ROP reduces the incidence of cataract development [7].

There are limited reports on the complications and visual outcomes of cataract surgery in children who have received treatment for ROP. Because these patients have many other vision limiting comorbidities, the presence of cataracts can pose a serious threat to the development of vision in these patients [7]. The purpose of our study is to report the surgical and visual outcomes of cataract surgery in pediatric patients who have had treatment for ROP.

\section{Methods}

Prior to initiating this study, institutional review board approval was obtained from the Baylor College of Medicine for the retrospective study. A comprehensive database search of our electronic medical record was performed to identify all patients treated for retinopathy of prematurity who had cataract surgery between August 2002 and March 2015. Inclusion criteria included patients with a history of ROP treatment with laser or intravitreal injection and who received cataract surgery with or without a lens implant for a subsequent cataract. Patients were excluded if they received lensectomy or cataract surgery at an outside institution.

The medical records were reviewed for details regarding ROP treatment, best corrected visual acuity (BCVA), slit lamp exam findings at each follow-up visit, intraoperative complications, ocular comorbidities, and adverse events. Subject parameters reviewed included sex, ethnicity, age at surgery, cataract laterality, and time of onset of cataract.

For patients unable to cooperate in clinic, intraocular lens calculations were performed in the operating room using immersion A-scan ultrasonography for axial length measurements and keratometry was obtained using a handheld keratometer. Cataract surgery was performed using standard technique [8]. Briefly, in cases in which the posterior capsule was left intact, a temporal clear corneal incision was made using a keratome and a continuous curvilinear capsulorrhexis was performed with utrata forceps; aspiration of the lens was accomplished using an automated irrigation/aspiration handpiece with the Accurus or Constellation system (Alcon Laboratories). In patients in which a primary posterior capsulotomy and anterior vitrectomy was planned, the anterior capsulotomy and lens aspiration were performed using a mechanical vitrectomy handpiece using 
bimanual technique. A primary posterior capsulotomy and anterior vitrectomy was then performed through the pars plana approach in a standardized fashion after placement of the intraocular lens.

All patients received a single piece, foldable acrylic IOL (Acrysof, SN60AT) if the IOL was placed in the bag and a 3-piece foldable acrylic IOL (Acrysof, MA60AC) if the IOL was placed in the sulcus. All patients were treated postoperatively with prednisolone acetate $1 \%$ eye drops every 2 hours for the first week followed by tapering over the next 4 weeks. Topical moxifloxacin was given 4 times a day for 1 week postoperatively.

Paired t-tests were used to compare visual outcomes before and after cataract surgeries, with significant difference defined as $p \leq 0.05$. BVCA was converted to logarithm of the minimum angle of resolution (LogMAR) before comparative statistics. Visual acuity described as fix and follow were excluded from comparisons of BCVA. Low vision described as blink to light (BTL) and light perception (LP) were converted to LogMAR 2.80, and no light perception (NLP) was converted to LogMAR 2.9 [9] [10] [11].

\section{Results}

A total of 19 eyes from 16 patients were identified which had cataract surgery due to development of cataracts after previously treated retinopathy of prematurity during the study time period. Average follow-up for the patients was 10.1 \pm 5.5 years (range: 1.5 years - 16.8 years). Ten eyes received cataract extraction with placement of an IOL (CE/IOL), 4 eyes received cataract extraction with anterior vitrectomy $(\mathrm{CE} / \mathrm{AntVx})$, and 5 eyes received cataract extraction with primary posterior capsulotomy and anterior vitrectomy (CE/IOL/PPC-AntVx) (Table 1). All of the patients received laser treatment for ROP except one; this

Table 1. Patient demographics.

\begin{tabular}{cc}
\hline Demographics & \\
Number of eyes & 19 eyes \\
Male (\%) & 10 patients $(37 \%)$ \\
Female (\%) & 6 patients $(63 \%)$ \\
Estimated Gestational Age & $24.8 \pm 2.0$ weeks \\
Birthweight & $747 \pm 233 \mathrm{~g}$ \\
Age at cataract surgery & $6.7 \pm 5.0$ years \\
Average length of follow up & $10.1 \pm 5.5$ years \\
Average age at time of surgery & \\
CE/IOL/PPC-AntVx (5 eyes, 26\%) & $3.3 \pm 1.7$ years \\
CE/IOL (10 eyes, 53\%) & $10.1 \pm 4.2$ years \\
CE/PPC-AntVx (4 eyes, 21\%) & $2.5 \pm 3.3$ years \\
Time between ROP treatment and cataract surgery & \\
Laser only (13 eyes, 69\%) & $6.2 \pm 5.6$ years \\
Laser + LSPPV (5 eyes, 26\%) & $6.4 \pm 4.2$ years \\
Intravitreal Bevacizumab (1 eye, 5\%) & 2.9 years \\
\hline
\end{tabular}

$\mathrm{CE}$, cataract extraction; IOL, intraocular lens; PPC, primary posterior capsulotomy; AntVx, anterior vitrectomy; LSPPV, lens sparing pars plana vitrectomy. 
patient received bilateral intravitreal injections of bevacizumab for bilateral stage 3 ROP with plus disease and developed a cataract in the left eye 3.4 months after the injections. The cataract became visually significant 2.9 years after receiving the injection. An isolated posterior capsular injury consistent with a needle entry into the lens was noted during the time of cataract extraction.

Fifteen of 19 eyes (79\%) had primary IOL implanted at the time of cataract extraction; two of 15 (13\%) of these patients had a sulcus fixated IOL placed with the rest having the IOL placed in the capsular bag. Four of 19 eyes (21\%) were initially left aphakic and three of four these aphakic eyes (75\%) were treated first with contact lenses and then received a secondary sulcus IOL at an average age of $2.2 \pm 0.7$ years (1.4 - 2.7 years). The fourth eye was intentionally left aphakic since biometry measurements showed that the patient would require a $-7.00 \mathrm{D}$ IOL to yield emmetropia.

Eleven of 19 eyes (58\%) were able to perform an objective visual acuity both before cataract surgery and at the most recent visit. In these 11 patients, vision improved from a preoperative BCVA of LogMAR $1.21 \pm 0.87$ (Snellen equivalent 20/324) to a post-operative BCVA of LogMar $0.74 \pm 0.77$ (Snellen equivalent 20/110) $(\mathrm{p}=0.06)$.

Two of 5 of the eyes (40\%) which had CE/IOL/PPC-Antvx and 8/10 (80\%) which had CE/IOL developed visually significant visual axis opacification (VAO) during the study period (Table 2). In patients who had CE/IOL/PPC-Antvx, the VAO involved lens reproliferation and in the patients who had CE/IOL, the VAO involved opacification of the posterior capsule. Six out of ten $(60 \%)$ of VAO-affected eyes required a surgical posterior capsulotomy due to the patient's inability to cooperate for a $\mathrm{Nd}$ :YAG laser capsulotomy while awake, either due to age or inability to fixate due to nystagmus. Two of the 6 (33\%) surgical posterior capsulotomy cases were performed by a vitreoretinal surgeon rather than by an anterior segment surgeon due to the concern of high risk for recurrent retinal detachment.

Intraoperative and post-operative complications are listed in Table 3. Iris hooks were required for visualization in three of the cases due to pupillary miosis. One eye developed acute angle-closure glaucoma two days postoperatively

Table 2. Visual axis opacification.

\begin{tabular}{ccc}
\hline & CE/IOL/PPC-AntVx & CE/IOL \\
\hline Number of eyes & 5 eyes (26\%) & 10 eyes (53\%) \\
Age at surgery & $3.3 \pm 1.7$ years & $10.1 \pm 4.2$ years \\
Eyes developing VAO & 2 eyes (40\%) & 8 eyes (80\%) \\
Time from CE to VS VAO & $388.5 \pm 232.6$ days & $911.3 \pm 979.7$ days \\
VAO treatment & Surgical PC-AntVx (2 eyes) & Surgical PC-AntVx (4 eyes) \\
& & Nd:YAG (4 eyes)
\end{tabular}

VAO, visual axis opacification; VS, visually significant; PC-AntVx, posterior capsulotomy and anterior vitrectomy; Nd:YAG, diode laser photocoagulation. 
Table 3. Intraoperative and postoperative complications.

\begin{tabular}{cc}
\hline$\underline{\text { Intraoperative }}$ & \\
Pupillary miosis & 3 eyes (16\%) \\
Posterior synechiae & 3 eyes (16\%) \\
Anterior capsular fibrosis & 3 eyes (16\%) \\
Posterior capsular rent & 4 eyes (21\%) \\
Postoperative & \\
Visual axis opacification & 10 eyes (53\%) \\
Pupillary membrane & 1 eyes (5\%) \\
Acute angle-closure glaucoma & 1 eye (5\%) \\
\hline
\end{tabular}

Complications during and after cataract surgery are listed for the 19 eyes included in the study.

due to an air bubble that became trapped in the posterior chamber; the angle closure resolved with emergent removal of the air bubble in the operating room and this patient has done well with final BCVA of 20/30. One eye developed a pupillary membrane after cataract surgery that required 2 surgical interventions to resolve. A posterior capsular rent was noted intraoperatively in four cases, and the IOLs were placed successfully in the bag with no postsurgical complications in 2 of the eyes. The remaining two patients received a sulcus IOL.

No patients developed a new or recurrent retinal detachment after cataract surgery in the study period. One patient who had a history of two LSPPV procedures developed phthisis over time and the vision declined to NLP 4.4 years after cataract surgery. Three of 19 eyes (16\%) developed corneal band keratopathy 1.85 years (range: 6 days - 5.5 years) after cataract surgery. Five of 19 eyes (26\%) had documented optic atrophy and 6/16 patients (38\%) had nystagmus. Seven patients (44\%) were treated for amblyopia. Seven patients (44\%) had strabismus; four of these patients were esotropic and three were exotropic. Two out of 7 of these patients (29\%) received strabismus surgery during the study time period.

\section{Discussion}

Cataracts can be challenging to treat in patients who have been treated for retinopathy of prematurity due to anatomic changes from previous laser or vitrectomy surgery [12]. The average incidence of pediatric cataracts in patients who have had ROP laser treatment ranges from $0.003 \%$ - 6\% [13] [14] [15] [16]. While sample size limited our analysis of laser parameters in eyes with cataracts compared to eyes without cataracts, the possible role of photocoagulation in cataractogenesis has been discussed in other studies [15] [16] [17]. Although the mechanism remains unknown, laser photocoagulation for treatment of retinopathy of prematurity has been proposed to cause cataracts through anterior segment bleeding and inflammation or thermal damage via energy absorption through persistent tunica vasculosa lentis. Some studies, however, have found no significant difference in the incidence of cataracts in ROP eyes that have tunica 
vasculosa lentis compared to the eyes that do not [16] [17]. Cataracts are a well-known long term complication of lens-sparing posterior vitrectomy and may occur in $15 \%$ - 19\% of children under 27 months of age after this surgery [18] [19].

Premature birth and ROP can also result in anatomical changes to the infant eye. Anterior segment changes in ROP treated patients may include a smaller eye, shorter axial length, a shallower anterior chamber, a more anteriorly inserted iris, and a thicker and more spherical lens [20] [21] [22] [23]. These changes can make cataract surgery challenging, especially due to the high elasticity of the pediatric anterior capsule and steepness of the anterior lens. This, in combination with an increased risk of zonular weakness and poor pupillary dilation, necessitates additional care in performing the anterior capsulorrhexis [8] [12] [24].

Anatomic changes from prior vitreoretinal surgery can make cataract extraction challenging as well. Prior pars plana vitrectomy can reduce posterior support which complicates anterior capsulorrhexis, causes occult zonular instability, and increases the risk of damage and instability to the posterior capsule [25]. Zonular weakness increases iris-lens-diaphragm mobility, thus leading to large fluctuations in the anterior chamber depth [26]. In our patient population, posterior capsular rent and instability was noted intraoperatively in 4 patients and led to placement of IOLs in the sulcus rather than in the bag in 2 of these patients.

Visually significant VAO is a common complication after pediatric cataract surgery and can be amblyogenic, occurring in up to $71 \%$ of pediatric patients who receive primary PPC-AntVx and virtually $100 \%$ of pediatric patients who have the capsule left intact [7]. This is consistent with our data in which $80 \%$ of the patients who had the capsule left intact required treatment for VAO within 2.5 years of the original cataract surgery. In young children or patients who are developmentally delayed, $\mathrm{PPC}-\mathrm{AntVx}$ is routinely performed due to a high risk of development of VAO soon after cataract surgery [7]; in our study, this procedure reduced the development of VAO from $80 \%$ to $40 \%$. If possible, we prefer to manage VAO with $\mathrm{Nd}$ :YAG laser capsulotomy, but many of our patients required a surgical posterior capsulotomy with anterior vitrectomy due to developmental delay causing inability to cooperate or, even when cooperation was good, nystagmus causing eye movement during the procedure. Due to the risk of retinal re-detachment, collaboration with a retinal surgeon may be required to perform the surgical posterior capsulotomy.

Other complications seen in our patient population included posterior synechiae. Because pediatric patients, in our experience, have greater postoperative inflammation, longer and more frequent use of topical steroids is often necessary after cataract surgery to reduce the incidence of synechiae.

The Early Treatment for Retinopathy of Prematurity Study found that $16 \%$ of the ROP patients who received laser only developed retinal detachment by 9 
months corrected age [27]. After lens-sparing vitrectomy (LSV) in children with stage 4 and 5 ROP, recurrent retinal detachment has been reported to be between $25 \%-73 \%$ of patients [19] [28]. Ezisi et al. noted retinal detachment to occur after cataract surgery in 3.5\% of their ROP patients treated by laser photocoagulation [12]. In our study, no patients developed a retinal detachment after surgery, but one patient with a history of treated retinal detachment did progress to phthisis 4.4 years after cataract extraction. Given the high rate of patients with ROP who develop retinal detachment after laser, the risk of recurrence is an important consideration.

The use of intravitreal bevacizumab has become a more frequent method of treatment for ROP [29]. Cataracts from mechanical lens injury due to intravitreal injections are rare but reported to occur in between $0 \%-0.07 \%$ of all patients who receive intravitreal injections [30] [31] [32]. In studies involving adult patients, Erdogan and colleagues found that iatrogenic lens injuries can progress to visually significant cataracts in $7 / 9(78 \%)$ of cases and can complicate surgery in $2 / 9$ (29\%) of cases due to posterior dislocation of lens fragments during phacoemulsification [33]. Modifying surgical technique is recommended to address the compromised capsular integrity [34]. In our patient who developed a cataract after an intravitreal bevacizumab injection, posterior capsule injury was noted in advance of cataract development. As the use of intravitreal bevacizumab increases in popularity for the treatment of ROP, the possibility of lens injury should be noted.

Our study was limited by its retrospective nature and small sample size. As a result, some data points were therefore not available. The young age or developmental status of our patient also limited our ability to quantify visual acuity in some patients.

Cataract surgery for children with history of ROP treatments presents many challenges. Prematurity and previous vitreoretinal surgeries may cause anterior segment changes that present intraoperative challenges such as miotic pupils, iris adhesions, a thicker lens, and capsular instability. Visual acuity can improve with cataract surgery but may be limited by other ocular comorbidities associated with prematurity. These patients need to be followed closely for the development of $\mathrm{VAO}$ and recurrence of retinal detachments.

\section{References}

[1] Farr, A.K., Stark, W.J. and Haller, J.A. (2001) Cataract Surgery by Phacoemulsification in Adults with Retinopathy of Prematurity. American Journal of Ophthalmology, 132, 306-310. https://doi.org/10.1016/S0002-9394(01)01006-6

[2] Davitt, B.V., Christiansen, S.P., Hardy, R.J., Tung, B. and Good, W.V. (2013) Early Treatment for Retinopathy of Prematurity Cooperative Group. Incidence of Cataract Development by 6 Months' Corrected Age in the Early Treatment for Retinopathy of Prematurity Study. Journal of AAPOS, 17, 49-53. https://doi.org/10.1016/j.jaapos.2012.10.011

[3] Holmes, J.M., Leske, D.A., Burke, J.P. and Hodge, D.O. (2003) Birth Prevalence of Visually Significant Infantile Cataract in a Defined U.S. Population. Ophthalmic 
Epidemiology, 10, 67-74. https://doi.org/10.1076/opep.10.2.67.13894

[4] Salvin, J.H., Lehman, S.S., Jin, J. and Hendricks, D.H. (2010) Update on Retinopathy of Prematurity: Treatment Options and Outcomes. Current Opinion in Ophthalmology, 21, 329-334. https://doi.org/10.1097/ICU.0b013e32833cd40b

[5] Mutlu, F.M. and Sarici, S.U. (2013) Treatment of Retinopathy of Prematurity: A Review of Conventional and Promising New Therapeutic Options. International Journal of Ophthalmology, 6, 228-236.

[6] Dahan, E. and Salmenson, B.D. (1990) Pseudophakia in Children: Precautions, Technique, and Feasibility. Journal of Cataract \& Refractive Surgery, 16, 75-82.

[7] Vasavada, A.R., Praveen, M.R., Tassignon, M.J., Shah, S.K., Vasavada, V.A., Vasavada, V.A., Van Looveren, J., De Veuster, I. and Trivedi, R.H. (2011) Posterior capsule Management in Congenital Cataract Surgery. Journal of Cataract \& Refractive Surgery, 37, 173-193.

[8] Pandey, S.K., Wilson, M.E., Trivedi, R.H., Izak, A.M., Macky, T.A., Werner, L. and Apple, D.J. (2001) Pediatric Cataract Surgery and Intraocular Lens Implantation: Current Techniques, Complications, and Management. International Ophthalmology Clinics, 41, 175-196.

[9] Roberts, M.F., Fishman, G.A., Roberts, D.K., Heckenlively, J.R., Weleber, R.G., Anderson, R.J. and Grover, S. (2002) Retrospective, Longitudinal, and Cross Sectional Study of Visual Acuity Impairment in Choroideraemia. British Journal of Ophthalmology, 86, 658-662. https://doi.org/10.1136/bjo.86.6.658

[10] Lee, J.W., Lai, J.S., Yick, D.W. and Tse, R.K. (2010) Retrospective Case Series on the Long-Term Visual and Intraocular Pressure Outcomes of Phacomorphic Glaucoma. Eye, 24, 1675-1680. https://doi.org/10.1038/eye.2010.108

[11] Schulze-Bonsel, K., Feltgen, N., Burau, H., Hansen, L. and Bach, M. (2006) Visual Acuities "Hand Motion" and "Counting Fingers" Can Be Quantified with the Freiburg Visual Acuity Test. Investigative Ophthalmology \& Visual Science, 47, 1236-1240. https://doi.org/10.1167/iovs.05-0981

[12] Ezisi, C.N., Kekunnaya, R., Jalali, S., et al. (2016) Cataract Surgery in Children with Retinopathy of Prematurity (ROP): Surgical Outcomes. British Journal of Ophthalmology, 2, 1128-1131.

[13] Fallaha, N., Lynn, M.J., Aaberg, T.M. and Lambert, S.R. (2002) Clinical Outcome of Confluent Laser Photoablation for Retinopathy of Prematurity. Journal of AAPOS, 6, 81-85. https://doi.org/10.1067/mpa.2002.121452

[14] Lambert, S.R., Capone, A., Cingle, K.A. and Drack, A.V. (2000) Cataract and Phthisis Bulbi after Laser Photoablation for Threshold Retinopathy of Prematurity. American Journal of Ophthalmology, 129, 585-591.

[15] Paysse, E.A., Miller, A., Brady McCreery, K.M. and Coats, D.K. (2002) Acquired Cataracts after Diode Laser Photocoagulation for Threshold Retinopathy of Prematurity. Ophthalmology, 109, 1662-1665.

[16] Christiansen, S.P. and Bradford, J.D. (1995) Cataract in Infants Treated with Argon Laser Photocoagulation for Threshold Retinopathy of Prematurity. American Journal of Ophthalmology, 119, 175-180.

[17] O’Neil, J.W., Hutchinson, A.K., Saunders, R.A. and Wilson, M.E. (1998) Acquired Cataracts after Argon Laser Photocoagulation for Retinopathy of Prematurity. Journal of AAPOS, 2, 48-51.

[18] Ferrone, P.J., Harrison, C. and Trese, M.T. (1997) Lens Clarity after Lens-Sparing 
Vitrectomy in a Pediatric Population. Ophthalmology, 104, 273-278.

[19] Choi, J., Kim, J.H., Kim, S.J. and Yu, Y.S. (2011) Long-Term Results of Lens-Sparing Vitrectomy for Stages 4B and 5 Retinopathy of Prematurity. Korean Journal of Ophthalmology, 25, 305-310. https://doi.org/10.3341/kjo.2011.25.5.305

[20] Chang, S.H.L., Lee, Y.S., Wu, S.C., See, L.C., Chung, C.C., Yang, M.L., Lai, C.C. and Wu, W.C. (2017) Anterior Chamber Angle and Anterior Segment Structure of Eyes in Children With Early Stages of Retinopathy of Prematurity. American Journal of Ophthalmology, 179, 46-54.

[21] Fledelius, H.C. (1992) Pre-Term Delivery and the Growth of the Eye. An Oculometric Study of Eye Size around Term-Time. Acta Ophthalmologica, 70, 10-15. https://doi.org/10.1111/j.1755-3768.1992.tb04915.x

[22] Fielder, A., Blencowe, H., O’Connor, A. and Gilbert, C. (2014) Impact of Retinopathy of Prematurity on Ocular Structures and Visual Functions. Archives of Disease in Childhood. Fetal and Neonatal Edition, 100, F179-F184.

[23] O’Connor, A.R., Wilson, C.M. and Fielder, A.R. (2007) Ophthalmological Problems Associated with Preterm Birth. Eye, 21, 1254-1260.

[24] Vasavada, A. and Chauhan, H. (1994) Intraocular Lens Implantation in Infants with Congenital Cataracts. Journal of Cataract \& Refractive Surgery, 20, 592-598.

[25] Shousha, M.A. and Yoo, S.H. (2010) Cataract Surgery after Pars Plana Vitrectomy. Current Opinion in Ophthalmology, 21, 45-49.

[26] Cole, C.J. and Charteris, D.G. (2009) Cataract Extraction after Retinal Detachment Repair by Vitrectomy: Visual Outcome and Complications. Eye, 23, 1377-1381. https://doi.org/10.1038/eye.2008.255

[27] Repka, M.X., Tung, B., Good, W.V., Shapiro, M., Capone, A., Baker, J.D., Barr, C.C., Phelps, D.L. and van Heuven, W.A. (2006) Outcome of Eyes Developing Retinal Detachment during the Early Treatment for Retinopathy of Prematurity Study (ETROP). Archives of Ophthalmology, 124, 24-30. https://doi.org/10.1001/archopht.124.1.24

[28] Yu, Y.S., Kim, S.-J., Kim, S.Y., Choung, H.K., Park, G.H. and Heo, J.W. (2006) Lens-Sparing Vitrectomy for Stage 4 and Stage 5 Retinopathy of Prematurity. Korean Journal of Ophthalmology, 20, 113-117.

https://doi.org/10.3341/kjo.2006.20.2.113

[29] Jager, R.D., Aiello, L.P., Patel, S.C. and Cunningham, E.T. (2004) Risks of Intravitreous Injection: A Comprehensive Review. Retina, 24, 676-698.

[30] Meyer, C.H., Rodrigues, E.B., Michels, S., Mennel, S., Schmidt, J.C., Helb, H.M., Hager, A., Martinazzo, M. and Farah, M.E. (2010) Incidence of Damage to the Crystalline Lens during Intravitreal Injectio ns. Journal of Ocular Pharmacology and Therapeutics, 26, 491-495. https://doi.org/10.1089/jop.2010.0045

[31] Khalifa, Y.M. and Pantanelli, S.M. (2011) Quiescent Posterior Capsule Trauma after Intravitreal Injection: Implications for the Cataract Surgeon. Journal of Cataract \& Refractive Surgery, 37, 1364.

[32] Fung, A.E., Rosenfeld, P.J. and Reichel, E. (2006) The International Intravitreal Bevacizumab Safety Survey: Using the Internet to Assess Drug Safety Worldwide. British Journal of Ophthalmology, 90, 1344-1349. https://doi.org/10.1136/bjo.2006.099598

[33] Erdogan, G., Gunay, B.O., Unlu, C., Gunay, M. and Ergin, A. (2016) Management of Iatrogenic Crystalline Lens Injury Occurred during Intravitreal Injection. Inter- 
national Ophthalmology, 36, 527-530. https://doi.org/10.1007/s10792-015-0156-5

[34] Saeed, M.U. and Prasad, S. (2009) Management of Cataract Caused by Inadvertent Capsule Penetration during Intravitreal Injection of Ranibizumab. Journal of Cataract \& Refractive Surgery, 35, 1857-1859. 